MIDAS

Museus e estudos interdisciplinares

$9 \mid 2018$

Varia

\title{
Competências para a transformação digital nos museus: o projecto $\mathrm{Mu}$.Sa
}

Competences for the digital transformation of museums: the Mu.Sa project

Ana Carvalho, Alexandre Matos e Manuel Morais Sarmento Pizarro

\section{(2) OpenEdition}

Journals

Edição electrónica

URL: http://journals.openedition.org/midas/1463

DOI: $10.4000 /$ midas. 1463

ISSN: 2182-9543

Editora:

Alice Semedo, Paulo Simões Rodrigues, Pedro Casaleiro, Raquel Henriques da Silva, Ana Carvalho

Refêrencia eletrónica

Ana Carvalho, Alexandre Matos e Manuel Morais Sarmento Pizarro, «Competências para a transformação digital nos museus: o projecto Mu.Sa », MIDAS [Online], 9 | 2018, posto online no dia 25 janeiro 2018, consultado no dia 01 maio 2019. URL : http://journals.openedition.org/midas/1463 ;

DOI : $10.4000 /$ midas. 1463

Este documento foi criado de forma automática no dia 1 Maio 2019.

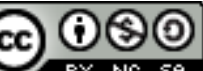

Midas is licensed under a Creative Commons Attribution-NonCommercial-ShareAlike 3.0 International License 


\section{Competências para a transformação digital nos museus: o projecto $\mathrm{Mu}$.Sa}

Competences for the digital transformation of museums: the Mu.Sa project

Ana Carvalho, Alexandre Matos e Manuel Morais Sarmento Pizarro

\section{Introdução'}

1 Vivemos numa sociedade cada vez mais tecnológica e digital. Sendo uma tendência mainstream é expectável que traga impactos também para os museus. Neste contexto, um longo caminho tem sido percorrido pelos museus ao longo das últimas décadas. Se numa fase inicial as novas tecnologias eram olhadas pelos profissionais de museus com reserva e desconfiança, passou-se, paulatinamente, para a sua crescente integração e para um papel mais activo da cultura digital nos museus contemporâneos (Parry 2013). Entre os argumentos que consubstanciam a reserva inicial - estávamos então no início da década de 1990 e um panorama museológico marcado pela limitação ou inexistência de competências digitais ou tecnológicas -, prevalecia o receio que as tecnologias pudessem reduzir o número de visitantes, que esbatessem a autenticidade da experiência de visita e o contacto com as colecções, ou pusessem em causa a transmissão do conhecimento científico (MacDonald 2006). Por outro lado, as novas tecnologias eram também vistas como dispositivos dispendiosos e de alto risco (Parry 2013).

2 No presente, uma maior implicação das tecnologias e do mundo digital no sector dos museus encontra outros argumentos. Entre estes, a ideia de que o mundo digital está cada vez mais entrosado na vida das pessoas, sobretudo dos mais jovens (nativos digitais), que no seu quotidiano aplicam já novas formas de aceder à informação. Neste sentido, para os museus, o universo do digital justifica-se pela oportunidade de acompanhar estes fenómenos, conquistar os públicos mais jovens e, por outro lado, a não ficar para trás num contexto cada vez mais competitivo quanto ao apelo do digital e do audiovisual. Outros argumentos sustentam que o mundo digital pode ajudar a democratizar os museus ao descentrar a tónica da autoridade, tradicionalmente remetida aos especialistas, para os visitantes, criando espaços para interpretações alternativas e mais plurais de objectos e 
iniciativas, ou possibilitar contextos mais favoráveis para que sejam os visitantes a dirigir a sua própria aprendizagem (Masson 2017). Sublinha-se, ainda, a possibilidade de se proporcionarem experiências mais flexíveis e criativas (Parry 2013).

Prevalece, assim, o reconhecimento, mais ou menos consensual, da necessidade de sintonização dos museus com novas formas de comunicação contemporâneas, tirando partido das tecnologias e do mundo digital para uma maior partilha de conhecimentos sobre as colecções, e estabelecer mais ligações à sociedade (Keene 2004). É também este argumento que encontramos plasmado na escolha do tema do Dia Internacional de Museus para 2018: Museus Hiperconectados: Novas Abordagens, Novos públicos. ${ }^{2}$

4 Tendo em conta as possibilidades que o desenvolvimento das tecnologias e do mundo digital oferecem em termos de novas soluções e aplicações, estes aportes podem ter implicações em pelo menos duas dimensões. Por um lado, na forma como os museus se organizam internamente, visando processos de gestão mais eficientes e ágeis (ex. gestão de colecções e arquivos, mas também de processos de trabalho). E, por outro lado, no modo como os museus comunicam o conhecimento produzido sobre as suas colecções, oferecendo experiências significativas, seja em contexto físico, seja em ambiente digital. Responder a estes desafios é, portanto, uma questão que atravessa todas as áreas de actuação dos museus (gestão, comunicação, educação, exposição, gestão de colecções e envolvimento de públicos), desde o trabalho de bastidores à frente de casa.

5 Todavia, a forma como cada museu responde a estes desafios é, porventura, muito diversa e desigual. A demanda de transformação para corresponder às expectativas dos novos tempos traz uma complexidade acrescida, o que implica uma reflexão informada sobre o lugar a dar às tecnologias e ao mundo digital, na perspectiva de instrumentos complementares de acesso e de transmissão, mas contextualizados e norteados pela missão e estratégia de cada museu. Esta demanda, que é, por um lado, do campo das ideias e da estratégia, é condicionada, por seu turno, pela existência ou não de um conjunto de meios que possa dar respostas em conformidade: recursos humanos, financeiros, técnicos e tecnológicos.

6 Este texto toma em consideração, em particular, a reflexão em torno da qualificação e actualização de competências dos profissionais de museus como um dos factores que pode contribuir para a transformação digital dos museus, uma preocupação que, aliás, tem acompanhado este tema (cf. Parry 2013).

7 Se tomarmos em consideração o Referencial Europeu das Profissões Museais (Ruge 2008), uma iniciativa do ICTOP (Comité Internacional do Conselho Internacional de Museus para a Formação de Pessoal), são identificadas 20 profissões no contexto do sector museológico europeu. Este referencial inclui uma descrição das funções de cada perfil, a formação de base necessária, a experiência complementar e um campo extra de observações. Entre as 20 profissões elencadas, destacam-se dois perfis cujas competências estão mais vocacionadas para o campo das tecnologias da informação e comunicação (TIC): o "responsável pelo sítio web" e o "responsável pelos sistemas informáticos". Acresce um terceiro perfil, de forma mais indirecta, o "responsável pelo inventário", que ao nível da formação prevê o «domínio das ferramentas informáticas» (Ruge 2008, 22).

8 Passados dez anos, e atendendo ao ritmo acelerado de adopção das TIC na sociedade contemporânea, e por seu turno nas organizações, de que forma é que esta transformação se tem reflectido na redefinição dos perfis profissionais? Que novas competências são necessárias? Que perfil de formação? Perspectivam-se novos perfis de profissionais? Que 
diagnóstico é possível fazer? Estas são algumas das interrogações que estão na génese do projecto Mu.Sa - Museum Sector Alliance (2016-2019) ${ }^{3}$. Neste breve artigo discutimos o racional do projecto, os seus objectivos e metodologias, assim como alguns dos resultados entretanto obtidos.

\section{O projecto Mu.Sa: objectivos e metodologias}

9 O projecto Mu.Sa - Museum Sector Alliance foi criado a pensar na escassez de competências digitais no sector dos museus, uma questão debatida no contexto de vários projectos europeus que o antecederam, nomeadamente o eCultskills - eskills for Future Cultural Jobs (2013-2015) ${ }^{4}$, e abordada frequentemente em reuniões e conferências de diferentes temáticas ligadas ao universo museológico nacional e internacional. Ainda que à maioria das profissões aplicadas ao trabalho de museu se exija um diploma de estudos universitários, a formação contínua e específica, ao longo da vida, continua a ser um aspecto fundamental para responder a novos campos de acção impulsionados pelas dinâmicas, exigências e expectativas da sociedade contemporânea (Ruge 2008; Horjan 2011). É neste contexto que se verifica a necessidade de dotar os profissionais de museus com competências que estimulem uma maior confiança e familiaridade relativamente aos desafios das TIC na sociedade contemporânea (Horjan 2011).

O projecto Mu.Sa tem como objectivo criar um conjunto de ferramentas educativas que promovam o desenvolvimento contínuo de competências digitais nos profissionais de museus, recorrendo a sistemas de formação de ensino à distância. A este propósito, o projecto inclui a concepção de um MOOC (Massive Open Online Courses), seguido de um curso de especialização.

11 O projecto tem por base um consórcio de 12 parceiros de quatro países europeus (Bélgica, Grécia, Itália e Portugal), sendo coordenado pela Hellenic Open University (Grécia). No caso português, incluem-se três parceiros: o ICOM Portugal ${ }^{5}$, a Universidade do Porto e a empresa Mapa das Ideias. Os restantes parceiros formam a seguinte composição - Melting Pro Learning (Itália), Link Campus University (Itália), National Organisation for the Certification of Qualifications and Vocational Guidance (Grécia), Symbola - Foundation for Italian Qualities (Itália), The Institute of Vocational Training AKMI (Grécia), Istituto per I Beni Artistici Culturali e Naturali della Regione Emilia Romagna (Itália), ICOM Grécia e Culture Action Europe (Bélgica).

o projecto organiza-se em duas fases distintas. A primeira fase, já concluída, consistiu no levantamento das necessidades dos profissionais de museus na área das tecnologias e do mundo digital, identificando perfis emergentes e competências, tendo por base uma análise de três contextos nacionais: Portugal, Grécia e Itália. Uma metodologia similar foi adoptada nos três países, entre Dezembro de 2016 e Março de 2017, através de vários métodos qualitativos: entrevistas, focus groups, inquéritos, além de pesquisa e revisão bibliográfica.

13 A segunda fase do projecto visa a elaboração de programas de formação (MOOC e curso de especialização), atendendo aos perfis profissionais emergentes e competências identificadas na primeira fase de mapeamento e pesquisa. Durante esta fase serão tidos em conta os standards de vários instrumentos europeus para que os resultados obtidos no projecto possam servir de referência comum a nível Europeu. Assim, os perfis profissionais e respectivas competências serão mapeados de acordo com a ESCO 
(Multilingual Classification of European Skills, Competences, Qualifications and Occupations), com o e-CF (European e-Competence Framework), incluindo a correspondência para o QNQ (Quadro Nacional de Qualificações) ${ }^{6}$ de Portugal, Grécia e Itália. Serão igualmente tidos em conta outros instrumentos de regulação no quadro europeu, tais como: o ECVET (European Credit System for Vocational Education and Training) e o EQAVET (European Quality Assurance in Vocational Education and Training).

14 Para cada um dos perfis identificados será desenvolvido um módulo curricular de Educação e Formação Profissional (EFP/VET), aplicando metodologias específicas baseadas em resultados de aprendizagem e princípios de educação de adultos focados em novas abordagens de educação e desenvolvimento de competências. É a partir deste trabalho que serão desenhados vários produtos de formação de ensino à distância, mas combinando sessões presenciais e aprendizagem em contexto de trabalho de museu.

A primeira etapa de formação a disponibilizar adopta o formato de MOOC em plataforma de código aberto (ex. edX). Com um perfil propedêutico, será composto por módulos de formação para a aquisição de competências básicas comuns aos perfis profissionais identificados, com uma duração entre seis a dez semanas. A escolha deste formato prende-se com a possibilidade de garantir, por um lado, um curso online disponível para um número ilimitado de formandos (em diferentes situações geográficas), de acesso livre e sem restrições, ou seja, não exigindo pré-requisitos de participação mas somente um registo. E, por outro lado, a oportunidade de ampliar conhecimentos e melhorar competências com vista a uma maior compreensão, familiaridade e confiança no campo dos desafios da transformação digital nos museus. $\mathrm{O}$ formato MOOC, que tem tido uma expansão significativa sobretudo a partir de 2012, além de consubstanciar uma nova forma de provisão de conteúdos online destinada a números massivos de formandos, com relativo sucesso e impacto, tem-se revelado na sua aplicação ao universo museológico igualmente proveitoso (cf. Mazolla 2015; Parry 2016).

O MOOC desenhado no âmbito do projecto Mu.Sa tem como público preferencial profissionais de museus em diferentes áreas e estudantes na área da Museologia (e áreas afins). Os formandos que completarem com êxito a primeira fase de formação (MOOC) poderão ser elegíveis para participar na segunda etapa de formação - o curso de especialização (25-30 formandos por cada país participante no projecto), mas para o qual acrescem critérios de selecção mais estritos, sendo privilegiados formandos com profissões no sector dos museus e/ou sector cultural, e com experiência de trabalho. 0 curso de especialização, com uma duração de aproximadamente seis meses, terá um perfil modular, tendo em conta as necessidades profissionais identificadas, e compreende uma componente teórica e prática. Tem por base o formato de ensino à distância, através de uma plataforma digital (ex. Moodle), disponibilizando conteúdos online e recursos educativos, mas prevê ainda aulas presenciais, actividades de auto-estudo e uma experiência de trabalho efectiva em museu (aproximadamente dez semanas).

17 Entre os objectivos do projecto Mu.Sa está a ampla disseminação dos programas de formação desenvolvidos e dos resultados da investigação produzida, através da publicação de relatórios, publicações e artigos, incluindo ainda presença na Internet ( http://www.project-musa.eu) e nas redes sociais (https://www.facebook.com/ MuseumSectorAlliance). É de notar, ainda, o envolvimento de especialistas externos nas discussões por via da organização de eventos públicos, onde se destaca a realização de várias conferências e debates em Portugal, na Grécia e em Itália, ao longo do projecto. ${ }^{7}$ 


\section{Mapeamento e resultados preliminares}

18

de museus na área das tecnologias e do mundo digital no contexto português, grego e italiano. Para esta análise foram preponderantes os resultados obtidos a partir da realização de entrevistas e de focus groups, nos quais participaram um total de 78 especialistas. A escolha dos participantes, tanto para as entrevistas como para os focus groups, teve em conta uma amostra diversa de realidades museológicas representadas, designadamente em termos de escala (museus nacionais, locais), tutela (pública e privada) e tipologias de museu. A diversidade do perfil dos participantes foi outro critério adoptado: profissionais que actuam em diferentes áreas dos museus, mas também especialistas, investigadores, entre outros colaboradores externos.

19 A partir das entrevistas e focus groups pretendeu-se compreender de que modo os museus estão a responder aos desafios das tecnologias e do mundo digital nas várias áreas de trabalho (gestão, comunicação, educação, exposição, gestão de colecções e envolvimento de públicos). Neste contexto a investigação evidenciou uma experiência muito fragmentada e pouco estruturada nos três países, que está relacionada com diferentes contextos, políticas, governança, competências e recursos investidos. Identificaram-se, por um lado, limitações em termos da desadequação de investimento em infraestruturas e outros recursos; e, por outro lado, a ausência de estratégias digitais nacionais no sector, assim como lideranças mais activas que possam tirar partido das oportunidades que oferece o mundo digital, promovendo estratégias nesse sentido. A necessidade de formação contínua no contexto das competências digitais foi um outro aspecto reconhecido.

20 A investigação visou, ainda, identificar perfis profissionais emergentes, assim como as competências digitais e transferíveis (hard skills e soft skills) ${ }^{8}$ que lhes estão associadas. Neste ponto confrontaram-se os resultados obtidos no projecto eCultSkills (antecedeu o projecto Mu.Sa), e que havia mapeado no contexto europeu cinco perfis profissionais emergentes (e respectivas competências) no âmbito do digital para a área da cultura: "Consultor Cultural para as TIC"; "Guia Cultural de TIC"; "Gestor de Activos Culturais Digitais"; "Gestor de Experiência Cultural Interactiva"; e "Gestor de Comunidade Cultural Online". O objectivo consistiu em verificar a relevância e actualidade destes perfis atendendo ao contexto museológico português, grego e italiano. Os resultados permitiram aferir quatro perfis profissionais emergentes no sector dos museus, com a seguinte ordem de prioridade: "Gestor da Estratégia Digital"; "Curador de Colecções Digitais; "Promotor de Experiências Digitais Interactivas"; e "Gestor de Comunidades Online". ${ }^{9}$ Além das competências específicas para cada perfil, identificaram-se competências (digitais e transferíveis) comuns aos quatro perfis (Silvaggi 2017). Igualmente partilhado entre os perfis, refira-se o conhecimento aprofundado sobre o funcionamento dos museus e a incorporação das funções e competências atribuídas a cada perfil no contexto do trabalho de equipa.

21 Adicionalmente, foram entrevistados 12 profissionais de museus reconhecidos internacionalmente no contexto europeu..$^{10}$ Nesta amostra, o perfil das organizações é preferencialmente o de museus de grande dimensão (equipas com mais de 50 profissionais) e de substancial envergadura em termos de recursos financeiros, o que permitiu, por um lado, alargar o escopo da análise e, por outro lado, suscitar uma reflexão 
prospectiva sobre os desafios actuais e as tendências esperadas no futuro, considerando o papel proeminente das tecnologias como catalisadoras de mudanças no sector. Entre as conclusões do relatório - Museum of the Future: Insights and Reflections from 10 International Museums -, sublinha-se: «[...] o museu do futuro será mais emocional; as pessoas terão a possibilidade de se conectar com os museus de diferentes maneiras, e o digital e o físico serão dimensões cada vez mais hiperconectadas, sendo dois lados da mesma moeda, tanto para os visitantes como para as equipas dos museus» (Sturabotti e Surace 2017, 8).

Uma outra dimensão analisada nos três países foi o mapeamento da formação na área da Museologia, quer no âmbito dos curricula académicos, quer no contexto da oferta de formação profissional contínua, procurando compreender de que modo se traduz a introdução das tecnologias e do mundo digital na formação dos profissionais de museus. Para esse efeito, foi realizado um inquérito online, complementado com pesquisa. Os dados obtidos não foram conclusivos para uma análise global e comparativa entre os três países, devido a problemas identificados na recolha da informação. Todavia, no caso português a informação colectada sugere que, tanto do ponto de vista dos curricula académicos (formação pós-graduada em Museologia), como da formação profissional contínua disponível para o sector dos museus, a abordagem às competências digitais revela-se insuficiente e limitada.

\section{Considerações finais}

23 A evolução das tecnologias e do mundo digital tem implicações em todos os aspectos do trabalho em museus, nomeadamente nas equipas. É neste ponto que se centra o projecto Mu.Sa: que competências digitais (e transferíveis) são necessárias para responder de forma equilibrada à necessidade de maior interligação entre o mundo físico e o digital, e manter os museus relevantes no séc. XXI? Como ficou patente neste artigo, a investigação conduzida aponta para a necessidade de repensar os perfis dos profissionais de museus e perspectiva a actualização das suas competências neste campo. No geral, o desenvolvimento de programas de formação contínua e as ferramentas educativas previstas no quadro do projecto Mu.Sa, em particular, podem contribuir para a capacitação das equipas dos museus e, consequentemente, ajudar os museus a abraçar a transformação digital com maior proficiência e agilidade, e de forma mais integrada.

Entre as limitações que esta análise convoca está a capacidade dos museus inserirem nas suas equipas novos perfis de profissionais. Com efeito, no âmbito do projecto identificaram-se quatro perfis: "Gestor da Estratégia Digital"; "Curador de Colecções Digitais"; "Promotor de Experiências Interactivas Digitais"; e "Gestor de Comunidades Online". O desafio consiste em ajustar as competências previstas nestes perfis aplicando-os em equipas pequenas (uma realidade evidenciada nos vários países), onde os profissionais acumulam funções de vários perfis por força dos constrangimentos de recursos humanos que são patentes na maioria dos museus dos países analisados. Outro aspecto a ressaltar é a reserva na generalização dos resultados da investigação para um universo mais alargado, em parte porque estes referem-se a contextos específicos dos três casos estudados. Por outro lado, a transformação digital que se ambiciona para os museus não está apenas dependente da revisão e actualização de competências dos profissionais de museus. Outros factores foram também evidenciados, sugerindo uma compreensão interligada e uma visão de conjunto. Referimo-nos à necessidade de políticas museológicas de estratégia nacional no campo das tecnologias e do mundo digital, e à 
disponibilização de recursos adequados (financeiros, infraestruturais, técnicos, tecnológicos). Identifica-se ainda o papel determinante das lideranças, a diferentes níveis, que exige uma postura mais atenta e proactiva, e a capacidade de abertura para gerir a mudança. O projecto Mu.Sa não visa responder a todas estas questões, mas através do mapeamento de alguns destes problemas poderá contribuir para o seu diagnóstico e uma reflexão em torno dos mesmos. Por outro lado, as próximas etapas do projecto, assentes em programas de formação, poderão perspectivar caminhos a aprofundar neste campo.

\section{Agradecimentos}

A investigação para este artigo foi desenvolvida e financiada no âmbito do projecto Mu.Sa - Museum Sector Alliance (575907-EEP-1-2016-1-EL-EPPKA2-SSA) que tem, por sua vez, o suporte da Comissão Europeia através do programa Erasmus + (Sector Skills Alliance). Os autores expressam o seu agradecimento aos parceiros portugueses envolvidos: ICOM Portugal, Mapa das Ideias e Universidade do Porto, assim como aos profissionais e especialistas que participaram nas actividades de investigação decorridas em Portugal.

\section{BIBLIOGRAFIA}

Horjan, Goranka. 2011. “Towards the Education we Really Need in Regional Museums.” In Staff and Training in Regional Museums, editado por Jane Legget, 13-15. Paris: ICOM International Committee for Regional Museums (ICR), International Commitee for the Training of Personnel (ICTOP).

Keene, Suzanne. 2004. “The Future of the Museum in the Digital Age.” ICOM News 3: 4.

MacDonald, Lindsay, ed. 2006. Digital Heritage Applying Digital Imaging to Cultural Heritage. Burlington: Elsevier.

Masson, Eef. 2017. "Media in Contemporary Heritage Preservation: The Case of the Amsterdam Museum." In Museums and Innovations, editado por Zvjezdana Antos, Annette B. Fromm, e Viv Golding, 163-74. Cambridge: Cambridge Scholars Publishing.

Mazzola, Lisa. 2015. "MOOCs and Museums: Not Such Strange Bedfellows." Journal of Museum Education 40 (2): 159-70.

Parry, Ross, Alex Moseley, Nichola Gretton, Rachel Tunstall e Matthew Mobbs. 2016. "Why MOOCs Matter: The Consequence of Massive Open Online Courses for Museums, Universities, and their Publics." MW2016: Museums and the Web 2016. http://mw2016.museumsandtheweb.com/paper/ why-moocs-matter-the-consequence-of-massive-open-online-courses-for-museums-universitiesand-their-publics/

Parry, Ross. 2013. “The Practice of Digital Heritage and the Heritage of Digital Practice.” In Museums in a Digital Age, 1-7. Leicester Readers in Museum Studies. Hoboken: Taylor and Francis.

Ruge, Angelika, ed. 2008. Referencial Europeu das Profissões Museais. [s.l.]: ICOM-ICTOP. http://icomportugal.org/multimedia/File/ReferencialPT.pdf 
Silvaggi, Antonia, ed. 2017. Museum Professionals in the Digital Era: Agents of Change and Innovation (Full Version Report). [s.1.]: Mu.Sa - Museum Sector Alliance. http://www.project-musa.eu/wpcontent/uploads/2017/03/MuSA-Museum-professionals-in-the-digital-era-full-version.pdf

Sturabotti, Domenico e Romina Surace, eds. 2017. Museum of the Future: Insights and Reflections from 10 International Museums. [s.l.]: Symbola Foundation e Mu.Sa project.

\section{NOTAS}

1. Os autores escrevem de acordo com a antiga ortografia.

2. http://network.icom.museum/international-museum-day/imd-2017/the-theme/ (consultado Janeiro 25, 2018).

3. O projecto Mu.Sa - Museum Sector Alliance (575907-EEP-1-2016-1-EL-EPPKA2-SSA) é financiado pela Comissão Europeia no âmbito do programa Erasmus + (http://www.project-musa.eu).

4. Projecto financiado pela Comissão Europeia que analisou a emergência de novos perfis profissionais no sector cultural em contexto europeu, tendo em consideração os standards do European Qualifications Framework (EQF) e do European e-Competence Framework (e-CF). Foi coordenado pela Hellenic Open University (Grécia). Cf. http://ecultskills-conference.eap.gr/? q=node/3 (consultado Janeiro 25, 2018).

5. Os autores deste artigo colaboram com a Comissão Nacional Portuguesa do Conselho Internacional de Museus (ICOM Portugal) na prossecução das actividades previstas por esta organização no âmbito do projecto Mu.Sa.

6. Note-se que o $Q N Q$, por sua vez, adopta os níveis de qualificação e respectivos descritores do EQF (European Qualifications Framework).

7. Entre as conferências internacionais já realizadas destacam-se as seguintes: 2nd International Conference: Digital Challenges for Museum Experts (Atenas, 25 de Novembro de 2016), Re-designing Museums: Digital Skills for Change and Innovation (Roma, 13 de Julho de 2017). Em Portugal foram organizados igualmente vários debates públicos: Piloting the MOOC. The Challenges of Digital Skills for Museum Sector. Identification of Needs Oriented to the Structure of Museum Professionals' Formation \& Training (Porto, 2 de Dezembro de 2016), Os Desafios do Digital para a Cultura e o Património (Barcarena, 10 de Novembro de 2017) e Desafios Digitais para os Museus: Perfis Profissionais, Tarefas, Competências e Formação (Porto, 11 de Dezembro de 2017).

8. Tomando como referência a definição apresentada pelo Dicionário online da Cambridge, por competências transferíveis referimo-nos àquelas competências são transversais a diferentes profissões ou carreiras. As competências transferíveis podem ser agrupadas em soft skills e hard skills. As soft skills dizem respeito a competências comportamentais de um profissional. Por exemplo, a liderança é considerada uma soft skill. As hard skills estão mais ligadas a competências de ordem técnica. Por exemplo, a proficiência na utilização de uma aplicação do MS Office pode ser apontada como uma hard skill. Todavia, no contexto do projecto Mu.sa, a análise relativamente às competências transferíveis dirigiu-se sobretudo às soft skills.

9. É de notar que a tradução para português dos quatro perfis é ainda preliminar nesta fase. As designações identificadas em língua inglesa são as seguintes: Digital Strategy Manager; Digital Collections Curator; Digital Interactive Experience Developer; e Online Community Manager.

10. Foram recolhidos testemunhos de profissionais de dez museus. Elencamos a lista dos museus representados seguindo a ordem e a terminologia usada no referido relatório: Hermitage (Rússia); Kiasma Museum of Contemporary Art (Finlândia); MAAT - Museum of Art, Architecture and Tecnology (Portugal); MUSE - Museo dell Scienze di Trento (Itália); Musée du Louvre (França); National Museum of Wales (País de Gales); Museo Nacional del Prado (Espanha); Polin - 
Museum of the History of Polish Jews (Polónia); Rijksmuseum (Holanda); e Victoria and Albert Museum (Inglaterra).

\section{RESUMOS}

Vivemos numa sociedade cada vez mais tecnológica e digital. Sendo uma tendência mainstream são expectáveis impactos também para o sector dos museus. De que forma é que esta transformação se tem reflectido na redefinição dos perfis dos profissionais de museus? Que novas competências são necessárias ou expectáveis? Há alterações ou actualizações a fazer? Perspectivam-se novos perfis de profissionais? Que perfil de formação? Que diagnóstico é possível fazer? Estas são algumas das interrogações que estão na génese do projecto Mu.Sa Museum Sector Alliance (2016-2019), um projecto apoiado pelo Programa Erasmus + que aborda a escassez de competências digitais e transferíveis nas profissões museais, através da construção de um conjunto de ferramentas educativas que promovam a formação profissional contínua. Neste pequeno artigo discutimos o racional do projecto, os seus objectivos e metodologias, assim como alguns dos resultados da investigação desenvolvida em Portugal, Grécia e Itália na primeira fase do projecto. Entre outros aspectos, a investigação evidenciou uma experiência muito fragmentada e pouco estruturada nos três países em termos de transformação digital, que se deve, em parte, a diferentes contextos (políticas, governança) e à limitação de investimento nos recursos necessários (financeiros, infraestruturais, técnicos, tecnológicos), incluindo os recursos humanos e o reforço das competências digitais nas equipas de museus de uma forma transversal. A necessidade de formação profissional contínua foi um outro aspecto reconhecido nos três países, tendo sido identificados quatro perfis profissionais emergentes no sector dos museus. 0 desenvolvimento de programas de formação no quadro do projecto Mu.Sa pode contribuir para a capacitação das equipas de museus e, consequentemente, ajudar os museus a abraçar a transformação digital com maior proficiência e agilidade.

We live in a society increasingly technological and digital. It is a mainstream tendency with inevitable impacts also for the museum sector. How is this transformation-affecting museum professional's profile roles? What new competences are necessary and expected? Are there changes or up-skilling to be made? What kind of professional training is needed? These are some of the questions that led to the development of project Mu.Sa - Museum Sector Alliance (2016-2019), a project funded by the Erasmus Plus Programme that directly addresses the shortage of digital and transferable skills in the museum sector, by supporting on-going professional development. In this short article, we discuss the project rationale, its objectives and methodologies, but also the key findings of the first phase of the research activities carried out in Portugal, Greece and Italy. Among other aspects, the research highlighted a very fragmented experience across the three countries in terms of the digital shift, partially determined by different contexts (policies, governance), and the availability of the necessary investment in resources (financial, logistical), including human resources and the strengthening of digital competences in museum professions across the whole organisation. On-going professional training for digital competences was also a need recognized in the three countries, and four emerging job profiles were identified. The development of training programmes in the framework of Mu.Sa project may contribute to empower museum's workforce and, consequently, help museums to achieve digital transformation with more proficiency and agility. 


\section{ÍNDICE}

Palavras-chave: competências digitais, profissionais de museus, tecnologias da informação e comunicação, formação contínua em museus, perfis profissionais emergentes

Keywords: digital competences, museum professionals, museum professional training, emerging job profiles, Mu.Sa - Museum Sector Alliance, information and communication technology

\section{AUTORES}

\section{ANA CARVALHO}

Com um percurso de mestrado e doutoramento na área da Museologia, actualmente é investigadora de pós-doutoramento no Centro Interdisciplinar de História, Culturas e Sociedades (CIDEHUS) da Universidade de Évora (Portugal). Publicou Museus e Diversidade Cultural: Da Representação aos Públicos (Direção-Geral do Património Cultural e Caleidoscópio, 2016), Os Museus e o Património Cultural Imaterial: Estratégias para o Desenvolvimento de Boas Práticas (Colibri, 2011), e organizou a publicação digital Participação: Partilhando a Responsabilidade (Acesso Cultura, 2016). Editora do boletim do ICOM Portugal e autora do blogue No Mundo dos Museus. Colabora como investigadora principal no projecto Mu.Sa - Museum Sector Alliance por parte do ICOM Portugal. Centro Interdisciplinar de História, Culturas e Sociedades (CIDEHUS) da Universidade de Évora, Palácio do Vimioso, Largo do Marquês de Marialva, n. 8 Apartado 94, 7000-809 Évora, Portugal, arcarvalho@uevora.pt

\section{ALEXANDRE MATOS}

Director do Departamento de Investigação e Formação da Sistemas do Futuro Lda. e Professor Afiliado da Faculdade de Letras da Universidade do Porto (Portugal). Doutor e mestre em Museologia pela Faculdade de Letras da Universidade do Porto e investigador do Centro de Investigação Transdisciplinar "Cultura, Espaço e Memória” (CITCEM) na mesma Universidade. Gestor do projecto Mu.Sa - Museum Sector Alliance por parte do ICOM Portugal.

Faculdade de Letras da Universidade do Porto, Via Panorâmica s/n, 4150-564 Porto, Portugal, ammatos@letras.up.pt

\section{MANUEL MORAIS SARMENTO PIZARRO}

Doutorando em Estudos do Património - Museologia (Faculdade de Letras da Universidade do Porto, Portugal). Mestre em Museologia (Faculdade de Ciências Sociais e Humanas da Universidade Nova de Lisboa). Colabora como investigador júnior no âmbito do projecto Mu.Sa Museum Sector Alliance por parte do ICOM Portugal. Investigador do Centro de Investigação Transdisciplinar “Cultura, Espaço e Memória” (CITCEM) da Universidade do Porto. Actualmente, exerce funções de assessor de Museologia e Património no Pelouro da Cultura da Câmara Municipal de Lisboa.

Faculdade de Letras da Universidade do Porto, Via Panorâmica s/n, 4150-564 Porto, Portugal, msarmentopizarro@gmail.com 